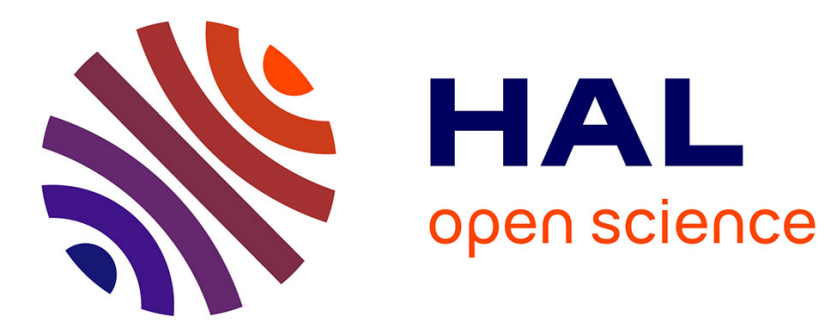

\title{
Barenblatt profiles for a nonlocal porous media equation
}

Piotr Biler, Cyril Imbert, Grzegorz Karch

\section{To cite this version:}

Piotr Biler, Cyril Imbert, Grzegorz Karch. Barenblatt profiles for a nonlocal porous media equation. Comptes rendus de l'Académie des sciences. Série I, Mathématique, 2011, 349, pp.641-645. 10.1016/j.crma.2011.06.003 . hal-00444392v2

\section{HAL Id: hal-00444392 \\ https://hal.science/hal-00444392v2}

Submitted on 6 Jun 2011

HAL is a multi-disciplinary open access archive for the deposit and dissemination of scientific research documents, whether they are published or not. The documents may come from teaching and research institutions in France or abroad, or from public or private research centers.
L'archive ouverte pluridisciplinaire HAL, est destinée au dépôt et à la diffusion de documents scientifiques de niveau recherche, publiés ou non, émanant des établissements d'enseignement et de recherche français ou étrangers, des laboratoires publics ou privés. 


\title{
Barenblatt profiles for a nonlocal porous medium equation \\ Piotr Biler ${ }^{\mathrm{a}}$, Cyril Imbert ${ }^{\mathrm{b}}$, Grzegorz Karch ${ }^{\mathrm{a}}$ \\ anstytut Matematyczny, Uniwersytet Wroctawski, pl. Grunwaldzki 2/4, 50-384 Wroctaw, Poland \\ ${ }^{\mathrm{b}}$ Université Paris-Dauphine, CEREMADE (UMR CNRS 7534), Place de Lattre de Tassigny, 75775 Paris Cedex 16, France \\ Received ... ; accepted after revision ... . \\ Presented by ...
}

\begin{abstract}
We study a generalization of the porous medium equation involving nonlocal terms. More precisely, explicit selfsimilar solutions with compact support generalizing the Barenblatt solutions are constructed. We also present a formal argument to get the $L^{p}$ decay of weak solutions of the corresponding Cauchy problem.

Résumé

Solutions auto-similaires pour une équation des milieux poreux non locale

Cette note est consacrée à l'étude d'une généralisation non locale de l'équation des milieux poreux. Plus précisément, on obtient des formules explicites de solutions auto-similaires à support compact qui ressemblent fortement aux solutions de type Barenblatt. On donne aussi un argument formel qui permet d'obtenir des estimations $L^{p}$ des solutions faibles du problème de Cauchy.
\end{abstract}

\section{Version française abrégée}

Nous considérons le problème de Cauchy pour l'équation non locale suivante

$$
\partial_{t} u-\nabla \cdot\left(u \nabla^{\alpha-1}\left(|u|^{m-1}\right)\right)=0,
$$

avec $m>1, x \in \mathbb{R}^{d}, \alpha \in(0,2), t>0$, à laquelle on ajoute une condition initiale $u(0, x)=u_{0}(x)$. Ici $\nabla^{\beta}$ est un opérateur intégral singulier généralisant le gradient usuel $(\beta=1)$ et lié au laplacien fractionnaire.

Le résultat principal de cette note sont des formules explicites de solutions auto-similaires qui se propagent à une vitesse finie.

\section{Théorème 1 (Solutions auto-similaires)}

La fonction

$$
u(t, x)=C t^{-\frac{d}{d(m-1)+\alpha}}\left(\left(R^{2}-|x|^{2} t^{-\frac{2}{d(m-1)+\alpha}}\right)_{+}^{\frac{\alpha}{2}}\right)^{\frac{1}{m-1}}
$$

(ainsi que ses translations en $x$ ) est une solution auto-similaire de l'équation (1) pour $R>0$ quelconque et une constante $C>0$ convenable.

Email addresses: biler@math.uni.wroc.pl (Piotr Biler), imbert@ceremade.dauphine.fr (Cyril Imbert), karch@math.uni.wroc.pl (Grzegorz Karch). 
Ensuite, nous présentons un calcul formel qui permet d'obtenir des estimations en norme $L^{p}\left(\mathbb{R}^{d}\right)$ des solutions faibles du problème de Cauchy, en particulier celles construites par Caffarelli et Vázquez [3] dans le cas $m=2$ et telle que $\left|u_{0}(x)\right| \leq C e^{-c|x|}$ pour deux constantes $C$ et $c$.

Calcul formel 1 (Asymptotique du problème de Cauchy)

Étant donnée une fonction $0 \leq u_{0} \in L^{1}\left(\mathbb{R}^{d}\right) \cap L^{\infty}\left(\mathbb{R}^{d}\right)$, les normes $L^{p}\left(\mathbb{R}^{d}\right), 1 \leq p<\infty$, des solutions faibles $u$ tendent vers 0 quand $t \rightarrow \infty$ avec le taux algébrique suivant

$$
\|u(t)\|_{p} \leq C(d, \alpha, m, p)\left\|u_{0}\right\|_{1}^{\frac{\alpha+d(m-1) / p}{\alpha+d(m-1)}} t^{-\frac{d}{d(m-1)+\alpha}(1-1 / p)} \quad \text { for all } t>0 .
$$

\section{Introduction}

We study a nonlocal generalization of the porous medium equation

$$
\partial_{t} u-\nabla \cdot\left(u \nabla^{\alpha-1}\left(|u|^{m-1}\right)\right)=0,
$$

where $m>1, \alpha \in(0,2), x \in \mathbb{R}^{d}, t>0$, supplemented with an initial condition

$$
u(0, x)=u_{0}(x) .
$$

The pseudodifferential (vector-valued) operator $\nabla^{\beta}$ in (1) is defined via the Fourier transform as $\nabla^{\beta} u=$ $\mathcal{F}^{-1}\left(i \xi|\xi|^{\beta-1} \mathcal{F} u\right)$. This definition is consistent with the usual gradient: $\nabla^{1}=\nabla$; the components of $\nabla^{0}$ are the Riesz transforms; moreover we have $\nabla \cdot \nabla^{\alpha-1}=\nabla^{\frac{\alpha}{2}} \cdot \nabla^{\frac{\alpha}{2}}=-(-\Delta)^{\frac{\alpha}{2}}$, where $(-\Delta)^{\frac{\alpha}{2}}$ denotes the fractional Laplace operator: $(-\Delta)^{\frac{\alpha}{2}} u=\mathcal{F}^{-1}\left(|\xi|^{\alpha} \mathcal{F} u\right)$. It can also be defined by real analysis tools as follows $\nabla^{\alpha-1} u=\nabla I_{2-\alpha} u$, where $I_{\beta}$ for $\beta \in(0, d)$ is an integral smoothing operator, called the Riesz potential (see $[12$, Ch. V])

$$
I_{\beta}(u)(x)=-C_{\beta} \int \frac{u(x+z)}{|z|^{d-\beta}} \mathrm{d} z
$$

with some $C_{\beta}>0$. Note that then $\nabla^{\alpha-1} u(x)=\nabla I_{2-\alpha}(u)(x)=(d+\alpha-2) C_{2-\alpha} \int(u(x+z)-u(x)) \frac{z}{|z|^{d+\alpha}} \mathrm{d} z$, $\alpha \in(0,2)$.

Eq. (1) can be interpreted as a transport equation of the type $\partial_{t} u=\nabla \cdot(u \mathbf{v})$ for some velocity vectorfield $\mathbf{v}$ which is a potential; more precisely, $\mathbf{v}=\nabla \mathbf{p}$ where $\mathbf{p}=I_{2-\alpha}\left(|u|^{m-1}\right)$. It can be interpreted as a nonlocal pressure in the case of nonnegative initial data. Then, of course $(-\Delta)^{\frac{2-\alpha}{2}} \mathbf{p}=|u|^{m-1}$, see [3], [4] for that notation.

Notice that for $\alpha=2$ and nonnegative initial data we recover the Boussinesq equation $(m=2)$, and the usual porous media equation $(m>1): \partial_{t} u=\nabla \cdot\left(u \nabla\left(u^{m-1}\right)\right), t>0, x \in \mathbb{R}^{d}$.

Recently, L. Caffarelli and J. L. Vázquez ([3], [4]) studied equation (1) in the case $m=2$. They proved the existence of weak solutions for nonnegative bounded integrable initial data with exponential decay at infinity. They also treat the case of bounded and compactly supported initial data, which propagate with finite speed. It is shown in [3] that self-similar solutions can be constructed by considering an obstacle problem for the fractional Laplace operator. In this note, we contribute to those results constructing explicit compactly supported self-similar solutions. Moreover, we show a kind of hypercontractivity estimates, i.e. the optimal decay in $L^{p}$ of general solutions with $u_{0} \in L^{1}\left(\mathbb{R}^{d}\right)$.

Results on equation (1) in this note are multidimensional generalizations of those obtained in [1] for a model of the dynamics of dislocations in crystals (for the integral of $u$ when $d=1$ and $m=2$ ). The structure of (1) suggests that it should enjoy the conservation of mass and some comparison properties as was shown in [1]. For an analysis of a related nonlocal equation, see [7].

Complete proofs of all results announced in this note will be published in [2].

\section{Self-similar solutions}

The equation (1) has the following scaling property: 
if $u(t, x)$ is a solution, so is $\ell^{d \lambda} u\left(\ell t, \ell^{\lambda} x\right)$

for each $\ell>0$ and $\lambda=\frac{1}{d(m-1)+\alpha}$. We look for nonnegative solutions that are invariant under that scaling, i.e. of the following form

$$
u(t, x)=\frac{1}{t^{d \lambda}} \Phi_{\alpha, m}\left(\frac{x}{t^{\lambda}}\right), \text { where } \quad \lambda=\frac{1}{d(m-1)+\alpha},
$$

for a function $\Phi_{\alpha, m}: \mathbb{R}^{d} \rightarrow \mathbb{R}^{+}$satisfying the following nonlinear and nonlocal equation in $\mathbb{R}^{d}$

$$
-\lambda \nabla \cdot\left(y \Phi_{\alpha, m}\right)=\nabla \cdot\left(\Phi_{\alpha, m} \nabla^{\alpha-1} \Phi_{\alpha, m}^{m-1}\right) .
$$

Before stating our main result, we recall the definition of weak solutions for (1) introduced in [3] in the case $m=2$.

Definition 2.1 (Weak solutions) A function $u:(0, T) \times \mathbb{R}^{d} \rightarrow \mathbb{R}$ is a weak solution of (1) in $Q_{T}=$ $(0, T) \times \mathbb{R}^{d}$ submitted to the initial condition $u(0, x)=u_{0}(x)$ if $u \in L^{1}\left(Q_{T}\right), I_{2-\alpha}\left(|u|^{m-1}\right) \in L^{1}\left(0, T ; W_{\text {loc }}^{1,1}\left(\mathbb{R}^{d}\right)\right)$, $u \nabla I_{2-\alpha}\left(|u|^{m-1}\right) \in L^{1}\left(Q_{T}\right)$ and

$$
\iint u\left(\varphi_{t}-\nabla I_{2-\alpha}\left(|u|^{m-1}\right) \cdot \nabla \varphi\right) \mathrm{d} x \mathrm{~d} t+\int u_{0}(x) \varphi(0, x) \mathrm{d} x=0
$$

for each test function $\varphi \in C^{1}\left(Q_{T}\right)$ such that $\varphi$ has compact support in the space variable $x$, and vanishes near $t=T$.

Theorem 2.2 (Self-similar solutions) For each $\alpha \in(0,2], m>1$ and $R>0$, the function

$$
\Phi_{\alpha, m}(y)=\left(k\left(R^{2}-|y|^{2}\right)_{+}^{\frac{\alpha}{2}}\right)^{\frac{1}{m-1}} \quad \text { with } \quad k=\left(\frac{d}{d(m-1)+\alpha}\right)\left(\frac{\Gamma\left(\frac{d}{2}\right)}{2^{\alpha} \Gamma\left(1+\frac{\alpha}{2}\right) \Gamma\left(\frac{d+\alpha}{2}\right)}\right)
$$

is a solution of (4). Consequently, the function

$$
u(t, x)=t^{-\frac{d}{d(m-1)+\alpha}}\left(k\left(R^{2}-|x|^{2} t^{-\frac{2}{d(m-1)+\alpha}}\right)_{+}^{\frac{\alpha}{2}}\right)^{\frac{1}{m-1}}
$$

is a weak solution of (1), satisfies the equation in the pointwise sense for $|x| \neq R t^{\frac{1}{d(m-1)+\alpha}}$, and is $\min \left\{\frac{\alpha}{2(m-1)}, 1\right\}$-Hölder continuous at the interface $|x|=R t^{\frac{1}{d(m-1)+\alpha}}$.

When $\alpha=2$, we recover the classical Kompaneets-Zel'dovich-Barenblatt-Pattle formulas, see, e.g., [13].

Note also that given $M>0$ there exists a unique $R>0$ such that $\int \Phi_{\alpha, m}(y) \mathrm{d} y=M$.

Remark 2.3 As mentioned above, self-similar solutions of (1) have been proved to exist in [3] by studying the following obstacle problem for the fractional Laplacian

$$
P \geq \Phi, \quad V=(-\Delta)^{\frac{\alpha}{2}} P \geq 0, \text { either } P=\Phi \text { or } V=0,
$$

with $\alpha \in(0,2)$ and $\Phi(y)=C-a|y|^{2}$. The novelty of our approach is that we exhibit the explicit solution of this obstacle problem: $P(y)=I_{\alpha}\left(\Phi_{\alpha, 2}\right)(y)$, where $I_{\alpha}$ is the Riesz potential, and $\Phi_{\alpha, 2}$ is defined in (6) with $m=2$ and a suitable $R>0$.

Those explicit self-similar solutions express one of the most important features of the porous medium equation: the property of finite propagation speed. In the case of the classical porous medium equation $(\alpha=2)$, this property is established using comparison with suitably large self-similar solutions, $c f$. [13]. For the generalized porous medium equation (1) with $m=2$, special supersolutions have been used for comparison, $c f .[4]$.

The proof of Theorem 2.2 is based on an application of the following fundamental technical fact. 
Lemma 2.4 For all $\beta \in(0,2)$ and $\gamma>0$, we have

$$
I_{\beta}\left(\left(1-|y|^{2}\right)_{+}^{\frac{\gamma}{2}}\right)= \begin{cases}C_{\gamma, \beta, d} \times{ }_{2} F_{1}\left(\frac{d-\beta}{2},-\frac{\gamma+\beta}{2} ; \frac{d}{2} ;|y|^{2}\right) & \text { for }|y| \leq 1, \\ \tilde{C}_{\gamma, \beta, d}|y|^{\beta-d} \times{ }_{2} F_{1}\left(\frac{d-\beta}{2}, \frac{2-\beta}{2} ; \frac{d+\gamma}{2} ; \frac{1}{|y|^{2}}\right) & \text { for }|y| \geq 1,\end{cases}
$$

with $C_{\gamma, \beta, d}=2^{-\beta} \frac{\Gamma\left(\frac{\gamma}{2}+1\right) \Gamma\left(\frac{d-\beta}{2}\right)}{\Gamma\left(\frac{d}{2}\right) \Gamma\left(\frac{\beta+\gamma}{2}+1\right)}$ and $\tilde{C}_{\gamma, \beta, d}=2^{-\beta} \frac{\Gamma\left(\frac{\gamma}{2}+1\right) \Gamma\left(\frac{d-\beta}{2}\right)}{\Gamma\left(\frac{d}{4}\right) \Gamma\left(\frac{d+\gamma}{2}+1\right)}$, where ${ }_{2} F_{1}$ is the hypergeometric function.

The verification of (8) consists in passing to Fourier transforms and calculating certain integrals (the so called (Sonine-)Weber-Schafheitlin integrals) involving Bessel and hypergeometric functions ${ }_{2} F_{1}, c f$. [11].

Proof of Theorem 2.2. Let $\phi_{\alpha}(y)=\left(1-|y|^{2}\right)_{+}^{\frac{\alpha}{2}}$. Observe that $\phi_{\alpha} \in L^{1}\left(\mathbb{R}^{d}\right)$. We next show that $I_{2-\alpha}\left(\phi_{\alpha}\right) \in$ $W_{\mathrm{loc}}^{1,1}\left(\mathbb{R}^{d}\right)$. By Lemma 2.4 with $\gamma=\alpha \in(0,2), \beta=2-\alpha$, and ${ }_{2} F_{1}(a,-1 ; c ; z)=1-\frac{a}{c} z$, we get

$$
I_{2-\alpha}\left(\phi_{\alpha}\right)(y)= \begin{cases}C_{\alpha, 2-\alpha, d}\left(1-\frac{d+\alpha-2}{d}|y|^{2}\right) & \text { if }|y| \leq 1, \\ \tilde{C}_{\alpha, 2-\alpha, d}|y|^{2-(d+\alpha)}{ }_{2} F_{1}\left(\frac{d+\alpha}{2}-1, \frac{\alpha}{2} ; \frac{d+\alpha}{2} ; \frac{1}{|y|^{2}}\right) & \text { if }|y| \geq 1,\end{cases}
$$

which is a locally integrable function. Recalling that $\nabla^{\alpha-1}=\nabla I_{2-\alpha}$, we then deduce by the chain rule that for $y \in B_{1}, \nabla^{\alpha-1}\left(\phi_{\alpha}\right)(y)=-\left(d K_{\alpha, d}\right)^{-1} y$ where $K_{\alpha, d}$ is defined in Corollary 2.5 below. For $|y| \geq 1$, one uses $\frac{\partial}{\partial z}{ }_{2} F_{1}(a, b ; c ; z)=\frac{a b}{c}{ }_{2} F_{1}(a+1, b+1 ; c+1 ; z)$, hence $\nabla^{\alpha-1}\left(\phi_{\alpha}\right)$ is locally integrable. To conclude, we remark that

$$
\phi_{\alpha}^{\frac{1}{m-1}}(y) \nabla^{\alpha-1} \phi_{\alpha}(y)=-\phi_{\alpha}^{\frac{1}{m-1}}(y)\left(K_{\alpha, d} d\right)^{-1} y, \text { for all } y \in \mathbb{R}^{d},
$$

because $\phi_{\alpha}(y)=0$ for $|y| \geq 1$.

Hence, scaling the variables, we immediately obtain that the function $\Phi_{\alpha, m}$ defined in (6) satisfies $\nabla^{\alpha-1}\left(\Phi_{\alpha, m}^{m-1}\right)=-\lambda y$ for $|y|<R$. Now, for all $y \in \mathbb{R}^{d}$ the identity

$$
-\lambda y \Phi_{\alpha, m}=\Phi_{\alpha, m} \nabla^{\alpha-1} \Phi_{\alpha, m}^{m-1}
$$

follows because $\Phi_{\alpha, m}(y)=0$ for all $|y| \geq R$, so (4) holds with $\lambda=\frac{1}{d(m-1)+\alpha}$ and $k=d \lambda K_{\alpha, d}$ defined in (6).

It is straightforward to verify using (4) that $u$ given by formula (7) is a weak solution of (1) in each strip $\left(t_{0}, T\right) \times \mathbb{R}^{d}, 0<t_{0}<T<\infty$. Moreover, the Hölder continuity is easy to check.

The following known result (with an important probabilistic interpretation) proved by Getoor [6, Th. $5.2]$, see also [9, App.] for a related calculation, is an immediate consequence of Lemma 2.4. For its proof, it suffices to use the relation $(-\Delta)^{\frac{\alpha}{2}}=\Delta I_{2-\alpha}$.

Corollary 2.5 For each $\alpha \in(0,2]$, the identity $K_{\alpha, d}(-\Delta)^{\frac{\alpha}{2}}\left(\left(1-|y|^{2}\right)_{+}^{\frac{\alpha}{2}}\right)=-1$ in $B_{1}$ holds with the explicit constant $K_{\alpha, d}=\frac{\Gamma\left(\frac{d}{2}\right)}{2^{\alpha} \Gamma\left(1+\frac{\alpha}{2}\right) \Gamma\left(\frac{d+\alpha}{2}\right)}$.

\section{The Cauchy problem and asymptotics}

We now briefly discuss the questions of the existence of weak solutions and their uniqueness in the case $m=2$. In [1], viscosity solutions have been considered which permitted the authors to prove regularity and uniqueness of solutions to (1) in one space dimension. In higher dimensions, a construction of mild solutions is achieved in [3] through a parabolic regularization of (1) with a suitable cutoff of the singular kernel of $I_{2-\alpha}$, and then a passage to the limit. The uniqueness of weak solutions and, a fortiori, the validity of the full comparison principle seem to be difficult questions, $c f$. a discussion in [3]. Another construction of weak solutions to (1) with $m>1$ as limits of mild solutions of parabolically perturbed equation (1) will be published in [2]. 
Formal computation 3.1 (Decay of solutions for the Cauchy problem) Suppose that for $0 \leq u_{0} \in$ $L^{1}\left(\mathbb{R}^{d}\right) \cap L^{\infty}\left(\mathbb{R}^{d}\right)$. Then $L^{p}\left(\mathbb{R}^{d}\right)$ norms $(1 \leq p<\infty)$ of any sufficiently regular and global in time nonnegative weak solution $u$ of (1)-(2) such that $\int u(t, x) \mathrm{d} x=\int u_{0}(x) \mathrm{d} x$ decay algebraically

$$
\|u(t)\|_{p} \leq C(d, \alpha, m, p)\left\|u_{0}\right\|_{1}^{\frac{\alpha+d(m-1) / p}{\alpha+d(m-1)}} t^{-\frac{d}{d(m-1)+\alpha}(1-1 / p)} \quad \text { for all } t>0 .
$$

Our computation below can be applied, for example, to weak solutions constructed by Caffarelli and Vázquez [3], who studied problem (1)-(2) with $m=2$ and with initial conditions satisfying $0 \leq u_{0}(x) \leq$ $C e^{-c|x|}$ for some constants $c, C$ and all $x \in \mathbb{R}^{d}$.

Formal proof of (9). In order to prove the announced $L^{p}$ estimates of solutions (similar to those for degenerated partial differential equations like the porous medium equation in e.g. [14], [5, Ch. 2]), we recall the Stroock-Varopoulos inequality for $q \geq 1$

$$
\int|w|^{q-2} w(-\Delta)^{\frac{\alpha}{2}} w \mathrm{~d} x \geq\left.\left.\frac{4(q-1)}{q^{2}} \int\left|\nabla^{\frac{\alpha}{2}}\right| w\right|^{\frac{q}{2}}\right|^{2} \mathrm{~d} x
$$

valid for each $w \in L^{q}\left(\mathbb{R}^{d}\right)$ such that $(-\Delta)^{\frac{\alpha}{2}} w \in L^{q}\left(\mathbb{R}^{d}\right)$. Note that the constant in (10) is the same as for the usual Laplacian operator $-\Delta$ (i.e. $\alpha=2$ ). The proof is given, e.g., in [10, Prop. 1.6] and [10, Th. 2.1 combined with (1.7)].

We will also need the following Nash inequality

$$
\|v\|_{2}^{2\left(1+\frac{\alpha}{d}\right)} \leq C_{N}\left\|\nabla^{\frac{\alpha}{2}} v\right\|_{2}^{2}\|v\|_{1}^{\frac{2 \alpha}{d}}
$$

valid for all functions $v$ with $v \in L^{1}\left(\mathbb{R}^{d}\right), \nabla^{\frac{\alpha}{2}} v \in L^{2}\left(\mathbb{R}^{d}\right)$ with a constant $C_{N}=C(d, \alpha)$. The proof of (11) for $d=1$ can be found in, e.g., [8, Lemma 2.2], and this extends easily to the general case $d \geq 1$.

Moreover, we will need the Gagliardo-Nirenberg type inequality: for $p>1$ and $p \geq m-1$,

$$
\|u\|_{p}^{a} \leq C_{N}\left\|\nabla^{\frac{\alpha}{2}}|u|^{\frac{r}{2}}\right\|_{2}^{2}\|u\|_{1}^{b}
$$

with $a=\frac{p}{p-1} \frac{d(r-1)+\alpha}{d}$ and $b=\frac{p \alpha+d(m-1)}{d(p-1)}$ and $r=p+m-1$. This inequality is a consequence of the Nash inequality (11) written for $v=|u|^{\frac{r}{2}}$, i.e. $\|u\|_{r}^{r\left(1+\frac{\alpha}{d}\right)} \leq C_{N}\left\|\nabla^{\frac{\alpha}{2}}|u|^{\frac{r}{2}}\right\|_{2}^{2}\|u\|_{\frac{r}{2}}^{\frac{r \alpha}{d}}$, and two Hölder inequalities for the $L^{q}$ norms: $\|u\|_{p} \leq\|u\|_{r}^{\gamma}\|u\|_{1}^{1-\gamma}$ and $\|u\|_{\frac{r}{2}} \leq\|u\|_{p}^{\delta}\|u\|_{1}^{1-\delta}$, which hold with $\gamma=\frac{p-1}{r-1} \frac{r}{p}$ and $\delta=\frac{r-2}{p-1} \frac{p}{r}$. Combining the above three inequalities, we get (12).

The computation, which lead to (9), consists in getting a differential inequality of the form $\frac{\mathrm{d}}{\mathrm{d} t} \int|u|^{p} \mathrm{~d} x \leq$ $-K\|u\|_{p}^{a}\|u\|_{1}^{-b}$ for some positive constant $K$ and where $a$ and $b$ appear in (12).

Multiply (1) by $u^{p-1}$ with $p>1$, integrate by parts, and use the relation $\nabla \cdot \nabla^{\alpha-1}=-(-\Delta)^{\frac{\alpha}{2}}$ to get

$$
\begin{aligned}
\frac{1}{p} \frac{\mathrm{d}}{\mathrm{d} t} \int u^{p} \mathrm{~d} x & =-(p-1) \int u^{p-1} \nabla u \cdot \nabla^{\alpha-1}\left(u^{m-1}\right) \mathrm{d} x \\
& =-\frac{p-1}{p} \int u^{p}(-\Delta)^{\frac{\alpha}{2}} u^{m-1} \mathrm{~d} x \\
& \leq-\frac{4(p-1)(m-1)}{(p+m-1)^{2}}\left\|\nabla^{\frac{\alpha}{2}}\left(u^{\frac{p+m-1}{2}}\right)\right\|_{2}^{2}
\end{aligned}
$$

after applying the Stroock-Varopoulos inequality (10) with $w=u^{m-1}$ and $q=\frac{p}{m-1}+1$. To estimate the right hand side of the above inequality, we use (12). We finally have:

$$
\frac{\mathrm{d}}{\mathrm{d} t} \int|u|^{p} \mathrm{~d} x \leq-K\|u\|_{p}^{a}\|u\|_{1}^{-b}
$$


with $K=\frac{1}{C_{N}} \frac{4 p(p-1)(m-1)}{(p+m-1)^{2}}$. The above inequality leads to the differential inequality $\frac{\mathrm{d}}{\mathrm{d} t} f(t) \leq-K M^{-b} f(t)^{\frac{a}{p}}$ for the function $f(t)=\|u(t)\|_{p}^{p}, M=\left\|u_{0}\right\|_{1}$, and $a / p>1$, which immediately gives the algebraic decay of the $L^{p}$ norms for $p \geq m-1: f(t) \leq\left(K\left(\frac{a}{p}-1\right) M^{-b} t\right)^{-\frac{1}{p}-1}$. Finally, we obtain the desired estimate with

$$
C(d, \alpha, m, p)=\left(K\left(\frac{a}{p}-1\right)\right)^{-\frac{1}{a-p}}=\left[\frac{4(m-1)(d(m-1)+\alpha)}{C_{N} d} \times \frac{p}{(p+m-1)^{2}}\right]^{-\frac{d}{d(m-1)+\alpha}\left(1-\frac{1}{p}\right)} .
$$

Of course, this is sufficient to get the conclusion of Theorem 3.1 for each $1 \leq p<\infty$ since $\frac{1}{\frac{a}{p}-1}=\frac{d(p-1)}{d(m-1)+\alpha}$, and interpolating between $L^{1}\left(\mathbb{R}^{d}\right)$ and $L^{p}\left(\mathbb{R}^{d}\right)$ with $p$ sufficiently large.

Remark 3.2 In our recent work [2], we present a more subtle iterative argument, which allows us to show that also

$$
\|u(t)\|_{\infty} \leq C\left\|u_{0}\right\|_{1}^{\frac{\alpha}{d(m-1)+\alpha}} t^{-\frac{d}{d(m-1)+\alpha}} \text { for all } t>0 .
$$

Acknowledgments. The preparation of this paper was supported by the Polish Ministry of Science (MNSzW) grant N201 418839, and a PHC POLONIUM project 0185, 2009-2010, and the Foundation for Polish Science operated within the Innovative Economy Operational Programme 2007-2013 funded by European Regional Development Fund (Ph.D. Programme: Mathematical Methods in Natural Sciences). The authors wish to thank Juan Luis Vázquez, Régis Monneau and Jean Dolbeault for fruitful discussions they had together.

\section{References}

[1] P. Biler, G. Karch and R. Monneau, A nonlinear diffusion of dislocation density and self-similar solutions, Commun. Math. Physics 294 (2010), 145-168.

[2] P. Biler, C. Imbert, G. Karch, Nonlocal porous medium equation: Barenblatt profiles and other weak solutions, in preparation, 2011.

[3] L. Caffarelli and J. L. Vázquez, Nonlinear porous medium flow with fractional potential pressure, preprint arXiv.1001.0410.v2, to appear in Arch. Rational Mech. Anal. DOI: 10.1007/s00205-011-0420-4.

[4] L. Caffarelli and J. L. Vázquez, Asymptotic behaviour of a porous medium equation with fractional diffusion, Discrete Cont. Dynam. Systems 29 (2011), 1393-1404.

[5] J. A. Carrillo, A. Jüngel, P. A. Markowich, G. Toscani and A. Unterreiter, Entropy dissipation methods for degenerate parabolic problems and generalized Sobolev inequalities, Monatsh. Math. 133, (2001), 1-82.

[6] R. K. Getoor, First passage times for symmetric stable processes in space, Trans. Amer. Math. Soc. 101 (1961), 75-90.

[7] C. Imbert and A. Mellet, A higher order non-local equation appearing in crack dynamics, preprint arXiv:1001.5105, 2010.

[8] G. Karch, C. Miao and X. Xu, On the convergence of solutions of fractal Burgers equation toward rarefaction waves, SIAM J. Math. Anal. 39 (2008), 1536-1549.

[9] N. S. Landkof, Foundations of Modern Potential Theory, Die Grundlehren der mathematischen Wissenschaften. Band 180. Berlin-Heidelberg-New York, Springer-Verlag, (1972).

[10] V. A. Liskevich and Yu. A. Semenov, Some problems on Markov semigroups, Schrödinger operators, Markov semigroups, wavelet analysis, operator algebras, 163-217, Math. Top. 11, Akademie Verlag, Berlin, (1996).

[11] W. Magnus, F. Oberhettinger, and R. P. Soni, Formulas and theorems for the special functions of mathematical physics, Third enlarged edition. Die Grundlehren der mathematischen Wissenschaften, Band 52, Springer-Verlag New York, Inc., New York, (1966).

[12] E. M. Stein, Singular Integrals and Differentiability Properties of Functions, Princeton University Press, Princeton, (1970).

[13] J. L. Vázquez, The Porous Medium Equation. Mathematical Theory, Oxford Mathematical Monographs; Oxford Science Publications. Oxford, Oxford University Press, (2007).

[14] J. L. Vázquez, Smoothing and Decay Estimates for Nonlinear Diffusion Equations. Equations of Porous Medium Type, Oxford Lecture Series in Mathematics and its Applications 33, Oxford University Press, Oxford, (2006). 